(C) 2012 IEEE. Personal use of this material is permitted. Permission from IEEE must be obtained for all other uses, in any current or future media, including reprinting/republishing this material for advertising or promotional purposes, creating new collective works, for resale or redistribution to servers or lists, or reuse of any copyrighted component of this work in other works. 


\title{
Interference MIMO Relay Channel: Joint Power Control and Transceiver-Relay Beamforming
}

Muhammad R. A. Khandaker, Student Member, IEEE, and Yue Rong, Senior Member, IEEE

\begin{abstract}
In this paper, we consider an interference multiple-input multiple-output (MIMO) relay system where multiple source nodes communicate with their desired destination nodes concurrently with the aid of distributed relay nodes all equipped with multiple antennas. We aim at minimizing the total source and relay transmit power such that a minimum signal-to-interference-plus-noise ratio (SINR) threshold is maintained at each receiver. An iterative joint power control and beamforming algorithm is developed to achieve this goal. The proposed algorithm exploits transmit-relay-receive beamforming technique to mitigate the interferences from the unintended sources in conjunction with transmit power control. In particular, we apply the semidefinite relaxation technique to transform the relay transmission power minimization problem into a semidefinite programming (SDP) problem which can be efficiently solved by interior point-based methods. Numerical simulations are performed to demonstrate the effectiveness of the proposed iterative algorithm.
\end{abstract}

\section{INTRODUCTION}

In a large wireless network with many nodes, multiple source-destination links must share a common frequency band concurrently to ensure a high spectral efficiency of the whole network [1]. In such network, cochannel interference $(\mathrm{CCI})$ is one of the main impairments that degrades the system performance. Developing schemes that mitigate the CCI is therefore important.

M. R. A. Khandaker and Y. Rong are with the Department of Electrical and Computer Engineering, Curtin University, Bentley, WA 6102, Australia (e-mail: m.khandaker@postgrad.curtin.edu.au; y.rong@ curtin.edu.au).

This work was supported under the Australian Research Council's Discovery Projects funding scheme (project numbers DP110100736, DP110102076). 
By exploiting the spatial diversity, multi-antenna technique provides an efficient approach to CCI minimization [1], [2]. When each source node has a single antenna and the destination nodes are equipped with multiple antennas, a joint power control and receiver beamforming scheme is developed in [3] to meet the signal-to-interference-plus-noise ratio (SINR) threshold with the minimal transmission power. A joint transmit-receive beamforming and power control algorithm is proposed in [4], when the source nodes also have multiple antennas. Due to the transmit diversity, the total transmit power required in [4] is less than that in [3].

In addition to the transmit and/or receive beamforming considered in [3] and [4], distributed/network beamforming technique [5] can further increase the reliability of the communication link even if the direct path between the transmitter and the receiver is subject to serious degradation, especially for long-distance communication. The network beamforming scheme stems from the idea of cooperative diversity [6]-[8], where users share their communication resources such as bandwidth and transmit power to assist each other in data transmission. The optimal relay matrix design has been recently studied for the multipleinput multiple-output (MIMO) broadcast channel [9] and the point-to-point MIMO relay channel [10], [11]. In [12], a decentralized relay beamforming technique has been developed considering a network of one transmitter, one receiver, and several relay nodes each having a single antenna. In [13], a wireless ad hoc network consisting of multiple source-destination pairs and multiple relay nodes, each having a single antenna, is considered, where the network beamforming scheme is used to meet the SINR threshold at all links with the minimal total transmission power consumed by all relay nodes. Relay beamformers are designed in [14] for multiple-antenna relay nodes with single-antenna source-destination pairs. The non-regenerative MIMO relay technique has been applied to multi-cellular (interference) systems in [15] where transceiver beamformers are designed using the partial zero-forcing (PZF) technique.

However, it is assumed in [13]-[15] that each source node uses its maximum available transmit power. Such assumption not only raises the system transmit power consumption, but also increases the interference from one user to all other users. This indicates that the beamforming and the power control problem should be considered jointly as in [3] and [4].

In this paper, we consider a two-hop interference MIMO relay system consisting of $L$ source-destination pairs communicating with the aid of $K$ relay nodes to enable successful communication over a long distance. Each of the source, relay and destination nodes is equipped with (possibly different number of) multiple antennas. The amplify-and-forward scheme is used at each relay node due to its practical implementation simplicity. In fact, these relay nodes assist in CCI mitigation by performing distributed 
network beamforming ${ }^{1}$.

We aim at developing a joint power control and beamforming algorithm such that the total transmission power consumed by all source nodes and relay nodes are minimized while maintaining the SINR at each receiver above a minimum threshold value. Compared with [12]-[14], we not only use the network beamforming technique at the relay nodes, but also apply the joint transmit-receive beamforming technique for multiple-antenna users to mitigate the CCI. In contrast to [15], we develop an iterative technique to solve the total power minimization problem rather than using the suboptimal PZF approach. Moreover, transmit power control is used in our algorithm to minimize the total transmit power and the interference to other users, which is not considered in [12]-[15].

A two-tier iterative algorithm is proposed to jointly optimize the source, relay and receive beamformers, and the source transmission power. We update the relay beamformer in the outer loop using fixed source power, transmit beamformers, and receive beamformers. Since the relay beamforming optimization problem is nonconvex, we use the semidefinite relaxation (SDR) technique to transform the problem into a semidefinite programming (SDP) problem which can be efficiently solved by interior point-based methods. Then in each iteration of the inner loop, we optimize the receive beamformers first with fixed transmit and relay beamformers and source power. Next, we update the source power such that the target SINR is just met with given transmit, relay and receive beamformers. Finally in the inner loop, we update the transmit beamformers with known transmit power, relay beamformers, and receive beamformers. Numerical simulations are carried out to evaluate the performance of the proposed algorithm.

The rest of this paper is organized as follows. In Section II, the system model of an interference MIMO relay network is introduced. The joint power control and beamforming algorithm is developed in Section III. Section IV shows the simulation results which justify the significance of the proposed algorithm under various scenarios. Conclusions are drawn in Section V.

\section{SySTEM MODEL}

We consider a two-hop interference MIMO relay system with $L$ source-destination pairs as illustrated in Fig. 1. Each source node communicates with its corresponding destination node with the aid of a network of $K$ distributed relays in order to enable successful communication over a long distance. The direct links between the source nodes and the destination nodes are not considered as they undergo much

\footnotetext{
${ }^{1}$ Although the relay beamforming matrices are optimized by a central processing unit in our algorithm, the relay beamforming operation is indeed distributed in the sense that the relays are geographically distributed and they perform beamforming only using their own received signal without exploiting the information on the received signals at other relay nodes.
} 
larger path attenuations compared with the links via relays. The source and destination nodes of the $l$ th link are equipped with $N_{\mathrm{s}, l}$ and $N_{\mathrm{d}, l}$ antennas, respectively, whereas the $k$ th relay node is mounted with $N_{\mathrm{r}, k}$ antennas.

We assume that all relay nodes work in half-duplex mode as in [12]-[14]. Thus the communication between the source-destination pairs is completed in two time slots. In the first time slot, the lth source node transmits an $N_{\mathrm{s}, l} \times 1$ signal vector $\mathbf{b}_{l} s_{l}$, where $s_{l}$ is the information-carrying symbol and $\mathbf{b}_{l}$ is the transmit beamforming vector. The received signal vector at the $k$ th relay node is given by

$$
\mathbf{y}_{\mathrm{r}, k}=\sum_{l=1}^{L} \mathbf{H}_{k, l} \mathbf{b}_{l} s_{l}+\mathbf{n}_{\mathrm{r}, k}, \quad k=1, \cdots, K
$$

where $\mathbf{H}_{k, l}$ is the $N_{\mathrm{r}, k} \times N_{\mathrm{s}, l}$ MIMO channel matrix between the $l$ th transmitting node and the $k$ th relay node and $\mathbf{n}_{\mathrm{r}, k}$ is the $N_{\mathrm{r}, k} \times 1$ additive Gaussian noise vector at the $k$ th relay node.

In the second time slot, the $k$ th relay node multiplies its received signal vector by an $N_{\mathrm{r}, k} \times N_{\mathrm{r}, k}$ complex matrix $\mathbf{F}_{k}$ and transmits the amplitude- and phase-adjusted version of its received signal. Thus the $N_{\mathrm{r}, k} \times 1$ signal vector $\mathbf{x}_{\mathrm{r}, k}$ transmitted by the $k$ th relay node is given by

$$
\mathbf{x}_{\mathrm{r}, k}=\mathbf{F}_{k} \mathbf{y}_{\mathrm{r}, k}, \quad k=1, \cdots, K .
$$

The received signal at the $l$ th destination node is obtained as the weighted sum of the received signals at each antenna element of that node, and is given by

$$
\begin{aligned}
y_{\mathrm{d}, l} & =\mathbf{w}_{l}^{H}\left(\sum_{k=1}^{K} \mathbf{G}_{l, k} \mathbf{x}_{\mathrm{r}, k}+\mathbf{n}_{\mathrm{d}, l}\right) \\
& =\mathbf{w}_{l}^{H}\left(\sum_{k=1}^{K} \mathbf{G}_{l, k} \mathbf{F}_{k}\left(\sum_{m=1}^{L} \mathbf{H}_{k, m} \mathbf{b}_{m} s_{m}+\mathbf{n}_{\mathrm{r}, k}\right)+\mathbf{n}_{\mathrm{d}, l}\right), \quad l=1, \cdots, L
\end{aligned}
$$

where $\mathbf{G}_{l, k}$ is the $N_{\mathrm{d}, l} \times N_{\mathrm{r}, k}$ MIMO channel matrix between the $k$ th relay node and the $l$ th destination node, $\mathbf{w}_{l}$ and $\mathbf{n}_{\mathrm{d}, l}$ are the $N_{\mathrm{d}, l} \times 1$ receive beamforming weight vector and the additive Gaussian noise vector at the $l$ th destination node, respectively, and $(\cdot)^{H}$ denotes matrix (or vector) Hermitian transpose. We assume that all noises are independent and identically distributed (i.i.d.) complex Gaussian noise with zero mean and variance $\sigma_{\mathrm{n}}^{2}$.

Let us introduce the following definitions

$$
\begin{aligned}
\tilde{\mathbf{h}}_{l} \triangleq\left[\left(\mathbf{H}_{1, l} \mathbf{b}_{l}\right)^{T}, \cdots,\left(\mathbf{H}_{K, l} \mathbf{b}_{l}\right)^{T}\right]^{T} \in \mathcal{C}^{\bar{N}_{\mathrm{r}} \times 1}, \quad l=1, \cdots, L \\
\tilde{\mathbf{G}}_{l} \triangleq\left[\mathbf{G}_{l, 1}, \cdots, \mathbf{G}_{l, K}\right] \in \mathcal{C}^{N_{\mathrm{d}, l} \times \bar{N}_{\mathrm{r}}}, \quad l=1, \cdots, L \\
\mathbf{F} \triangleq \operatorname{blkdiag}\left(\mathbf{F}_{1}, \mathbf{F}_{2}, \cdots, \mathbf{F}_{K}\right) \in \mathcal{C}^{\bar{N}_{\mathrm{r}} \times \bar{N}_{\mathrm{r}}} \\
\tilde{\mathbf{n}}_{\mathrm{r}} \triangleq\left[\mathbf{n}_{\mathrm{r}, 1}^{T}, \cdots, \mathbf{n}_{\mathrm{r}, K}^{T}\right]^{T} \in \mathcal{C}^{\bar{N}_{\mathrm{r}} \times 1}
\end{aligned}
$$


where $(\cdot)^{T}$ denotes matrix (or vector) transpose, blkdiag $(\cdot)$ stands for a block-diagonal matrix, and $\bar{N}_{\mathrm{r}} \triangleq \sum_{k=1}^{K} N_{\mathrm{r}, k}$. Here $\tilde{\mathbf{h}}_{l}$ can be viewed as the effective first-hop channel vector between $s_{l}$ and all relay nodes, $\tilde{\mathbf{G}}_{l}$ is the MIMO channel matrix between all relay nodes and the $l$ th receiver, $\mathbf{F}$ is the effective block-diagonal relay precoding matrix, and $\tilde{\mathbf{n}}_{\mathrm{r}}$ is a vector containing the noises at all relay nodes. Using these definitions, (2) can be rewritten as

$$
\begin{aligned}
y_{\mathrm{d}, l} & =\mathbf{w}_{l}^{H} \tilde{\mathbf{G}}_{l} \mathbf{F}\left(\sum_{m=1}^{L} \tilde{\mathbf{h}}_{m} s_{m}+\tilde{\mathbf{n}}_{r}\right)+\mathbf{w}_{l}^{H} \mathbf{n}_{\mathrm{d}, l} \\
& =\mathbf{w}_{l}^{H}\left(\sum_{m=1}^{L} \boldsymbol{\psi}_{m l} s_{m}+\mathbf{n}_{l}\right), \quad l=1, \cdots, L
\end{aligned}
$$

where $\boldsymbol{\psi}_{m l} \triangleq \tilde{\mathbf{G}}_{l} \mathbf{F} \tilde{\mathbf{h}}_{m}$ is the equivalent vector channel response between the $m$ th source node and the $l$ th destination node, and $\mathbf{n}_{l} \triangleq \tilde{\mathbf{G}}_{l} \mathbf{F} \tilde{\mathbf{n}}_{r}+\mathbf{n}_{\mathrm{d}, l}$ is the equivalent noise vector at the $l$ th receiver.

From (3), the total power of the received signal at the destination node of the $l$ th link is given by

$$
\mathrm{E}\left\{y_{\mathrm{d}, l} y_{\mathrm{d}, l}^{*}\right\}=\sum_{m=1}^{L} p_{m} \mathbf{w}_{l}^{H} \boldsymbol{\psi}_{m l} \boldsymbol{\psi}_{m l}^{H} \mathbf{w}_{l}+\mathbf{w}_{l}^{H} \mathbf{C}_{l} \mathbf{w}_{l}, \quad l=1, \cdots, L
$$

where $\mathrm{E}\{\cdot\}$ stands for statistical expectation, $(\cdot)^{*}$ denotes complex conjugate, $\mathbf{C}_{l} \triangleq \sigma_{\mathrm{n}}^{2} \tilde{\mathbf{G}}_{l} \mathbf{F} \mathbf{F}^{H} \tilde{\mathbf{G}}_{l}^{H}+$ $\sigma_{\mathrm{n}}^{2} \mathbf{I}_{N_{\mathrm{d}, l}}$ is the covariance matrix of $\mathbf{n}_{l}$, and $\mathbf{I}_{n}$ is an $n \times n$ identity matrix. Here we assume that $\mathrm{E}\left\{\left|s_{l}\right|^{2}\right\}=$ $p_{l}$ is the transmit power of the $l$ th information-carrying symbol. Based on (4), the SINR at the $l$ th destination node is given by

$$
\Gamma_{l}=\frac{p_{l} \mathbf{w}_{l}^{H} \boldsymbol{\psi}_{l l} \boldsymbol{\psi}_{l l}^{H} \mathbf{w}_{l}}{\sum_{m \neq l}^{L} p_{m} \mathbf{w}_{l}^{H} \boldsymbol{\psi}_{m l} \boldsymbol{\psi}_{m l}^{H} \mathbf{w}_{l}+\mathbf{w}_{l}^{H} \mathbf{C}_{l} \mathbf{w}_{l}}, \quad l=1, \cdots, L .
$$

Using (1), the transmission power consumed by the $k$ th relay node can be expressed as

$$
P_{\mathrm{r}, k}=\operatorname{tr}\left(\mathrm{E}\left\{\mathbf{x}_{\mathrm{r}, k} \mathbf{x}_{\mathrm{r}, k}^{H}\right\}\right)=\operatorname{tr}\left(\mathbf{F}_{k} \mathbf{R}_{\mathrm{y}, k} \mathbf{F}_{k}^{H}\right), \quad k=1, \cdots, K
$$

where $\operatorname{tr}(\cdot)$ denotes matrix trace, $\mathbf{R}_{\mathrm{y}, k} \triangleq \mathrm{E}\left\{\mathbf{y}_{\mathrm{r}, k} \mathbf{y}_{\mathrm{r}, k}^{H}\right\}=\sum_{l=1}^{L} p_{l} \mathbf{H}_{k, l} \mathbf{b}_{l} \mathbf{b}_{l}^{H} \mathbf{H}_{k, l}^{H}+\sigma_{\mathrm{n}}^{2} \mathbf{I}_{N_{\mathrm{r}, k}}$ is the covariance matrix of the received signal vector at the $k$ th relay node. Using (6), the total transmit power consumed by the whole network can be expressed as

$$
P_{\mathrm{T}}=\sum_{k=1}^{K} P_{\mathrm{r}, k}+\sum_{l=1}^{L} p_{l} \mathbf{b}_{l}^{H} \mathbf{b}_{l}
$$

\section{Joint Power Control And BeAmforming}

Let us define the relay beamforming vector $\mathbf{f}$ from the relay amplifying matrices $\mathbf{F}_{1}, \cdots, \mathbf{F}_{K}$ as

$$
\mathbf{f}=\left[\begin{array}{c}
\mathbf{f}_{1} \triangleq \operatorname{vec}\left(\mathbf{F}_{1}\right) \\
\vdots \\
\mathbf{f}_{K} \triangleq \operatorname{vec}\left(\mathbf{F}_{K}\right)
\end{array}\right] \in \mathcal{C}^{\tilde{N}_{\mathrm{r}} \times 1}
$$


where $\tilde{N}_{\mathrm{r}} \triangleq \sum_{k=1}^{K} N_{\mathrm{r}, k}^{2}$, and $\operatorname{vec}(\cdot)$ stands for a vector obtained by stacking all column vectors of a matrix on top of each other. In this section, we design the source transmit power vector $\mathbf{p} \triangleq\left[p_{1}, p_{2}, \cdots, p_{L}\right]^{T}$, the relay beamforming vector $\mathbf{f}$, transmit beamforming vectors $\left\{\mathbf{b}_{l}\right\} \triangleq\left\{\mathbf{b}_{l}, l=1, \cdots, L\right\}$, and receive beamforming vectors $\left\{\mathbf{w}_{l}\right\} \triangleq\left\{\mathbf{w}_{l}, l=1, \cdots, L\right\}$, such that a target SINR threshold $\gamma_{l}>0, l=1, \cdots, L$, is maintained at the $l$ th destination node with the minimal $P_{\mathrm{T}}$. The optimization problem can be written as

$$
\begin{aligned}
\min _{\mathbf{p}, \mathbf{f},\left\{\mathbf{b}_{l}\right\},\left\{\mathbf{w}_{l}\right\}} & P_{\mathrm{T}} \\
\text { s.t. } & \Gamma_{l} \geq \gamma_{l}, \quad l=1, \cdots, L .
\end{aligned}
$$

The problem (9)-(10) is nonconvex due to the constraints in (10). We propose a two-tier iterative algorithm to efficiently solve the problem (9)-(10). In the following, we solve corresponding subproblems to optimize each variable.

\section{A. Receive Beamforming}

The optimal receive beamforming vectors $\mathbf{w}_{l}, l=1, \cdots, L$, for fixed $\mathbf{p}, \mathbf{f}$, and $\left\{\mathbf{b}_{l}\right\}$ can be obtained such that it minimizes the noise-plus-interference power at the receiver under the condition of unity gain for the signal of interest, which can be written as

$$
\begin{aligned}
\min _{\mathbf{w}_{l}} & \sum_{m \neq l}^{L} p_{m} \mathbf{w}_{l}^{H} \boldsymbol{\psi}_{m l} \boldsymbol{\psi}_{m l}^{H} \mathbf{w}_{l}+\mathbf{w}_{l}^{H} \mathbf{C}_{l} \mathbf{w}_{l} \\
\text { s.t. } & \mathbf{w}_{l}^{H} \boldsymbol{\psi}_{l l}=1 .
\end{aligned}
$$

The unity gain condition ensures that the desired signal is unaffected by beamforming. Using the Lagrangian multiplier method, the solution to the problem (11)-(12) is given by

$$
\mathbf{w}_{l}=\frac{\boldsymbol{\Phi}_{l}^{-1} \boldsymbol{\psi}_{l l}}{\boldsymbol{\psi}_{l l}^{H} \boldsymbol{\Phi}_{l}^{-1} \boldsymbol{\psi}_{l l}}
$$

where $\mathbf{\Phi}_{l} \triangleq \sum_{m \neq l}^{L} p_{m} \boldsymbol{\psi}_{m l} \boldsymbol{\psi}_{m l}^{H}+\mathbf{C}_{l}$ is the interference-plus-noise covariance matrix at the $l$ th receiver, and $(\cdot)^{-1}$ denotes matrix inversion.

\section{B. Transmit Power Allocation}

To obtain optimal $\mathbf{p}$ with given beamforming vectors $\mathbf{f},\left\{\mathbf{b}_{l}\right\}$, and $\left\{\mathbf{w}_{l}\right\}$, we reformulate the problem (9)-(10) as

$$
\begin{array}{ll}
\min _{\mathbf{p}} & P_{\mathrm{T}} \\
\text { s.t. } & \frac{p_{l}[\overline{\mathbf{H}}]_{l, l}}{\sum_{m \neq l}^{L} p_{m}[\overline{\mathbf{H}}]_{m, l}+\bar{n}_{l}} \geq \gamma_{l}, \quad l=1, \cdots, L
\end{array}
$$


where $\overline{\mathbf{H}}$ is an $L \times L$ covariance matrix such that $[\overline{\mathbf{H}}]_{m, l}=\mathbf{w}_{l}^{H} \boldsymbol{\psi}_{m l} \boldsymbol{\psi}_{m l}^{H} \mathbf{w}_{l}$ and $\bar{n}_{l} \triangleq \mathbf{w}_{l}^{H} \mathbf{C}_{l} \mathbf{w}_{l}$. Here for a matrix $\mathbf{A},[\mathbf{A}]_{i, j}$ indicates the $(i, j)$ th element of $\mathbf{A}$. In the optimal power allocation, the transmit power of each user is set to the minimum required level such that the target SINR is just met [3], [4]. That is, the constraints in (15) should hold with equality as

$$
\frac{p_{l}[\overline{\mathbf{H}}]_{l, l}}{\sum_{m \neq l}^{L} p_{m}[\overline{\mathbf{H}}]_{m, l}+\bar{n}_{l}}=\gamma_{l}, \quad l=1, \cdots, L
$$

which can be equivalently rewritten as

$$
p_{l}=\frac{\gamma_{l}}{[\overline{\mathbf{H}}]_{l, l}}\left(\sum_{m \neq l}^{L} p_{m}[\overline{\mathbf{H}}]_{m, l}+\bar{n}_{l}\right), \quad l=1, \cdots, L
$$

Equation (17) can be written in matrix form as

$$
\mathbf{p}=\breve{\mathbf{H}} \mathbf{p}+\mathbf{u}
$$

where $[\breve{\mathbf{H}}]_{l, m}=\left\{\begin{array}{ll}0, & m=l \\ \gamma_{l}[\overline{\mathbf{H}}]_{m, l} /[\overline{\mathbf{H}}]_{l, l}, & m \neq l\end{array}\right.$, and $\mathbf{u}$ is an $L \times 1$ vector whose $l$ th element is given by $\gamma_{l} \bar{n}_{l} /[\overline{\mathbf{H}}]_{l, l}, l=1, \cdots, L$. From (18), it can be seen that the optimal solution to the problem (14)-(15) is given by

$$
\mathbf{p}=\left(\mathbf{I}_{L}-\breve{\mathbf{H}}\right)^{-1} \mathbf{u}
$$

\section{Transmit Beamforming}

With given $\mathbf{p}, \mathbf{f}$ and $\left\{\mathbf{w}_{l}\right\}$, the optimal $\left\{\mathbf{b}_{l}\right\}$ can be obtained simply by swapping the roles of the transmitters and the receivers as in [16]. First we rewrite the objective function by substituting $P_{\mathrm{r}, k}$ in (6) into (7) as

$$
P_{\mathrm{T}}=\sum_{l=1}^{L} p_{l}\left[\sum_{k=1}^{K} \operatorname{tr}\left(\mathbf{Q}_{k, l} \mathbf{b}_{l} \mathbf{b}_{l}^{H}\right)+\mathbf{b}_{l}^{H} \mathbf{b}_{l}\right]+\sigma_{\mathrm{n}}^{2} \sum_{k=1}^{K} \operatorname{tr}\left(\mathbf{F}_{k} \mathbf{F}_{k}^{H}\right)
$$

where $\mathbf{Q}_{k, l} \triangleq \mathbf{H}_{k, l}^{H} \mathbf{F}_{k}^{H} \mathbf{F}_{k} \mathbf{H}_{k, l}$. Let us now denote $\mathbf{Q}_{l} \triangleq \sum_{k=1}^{K} \mathbf{Q}_{k, l}$ and $\tilde{\mathbf{b}}_{l} \triangleq\left(\mathbf{Q}_{l}+\mathbf{I}_{N_{\mathrm{s}, l}}\right)^{\frac{1}{2}} \mathbf{b}_{l}$. Thus (20) can be equivalently written as

$$
P_{\mathrm{T}}=\sum_{l=1}^{L} p_{l} \tilde{\mathbf{b}}_{l}^{H} \tilde{\mathbf{b}}_{l}+\sigma_{\mathrm{n}}^{2} \sum_{k=1}^{K} \operatorname{tr}\left(\mathbf{F}_{k} \mathbf{F}_{k}^{H}\right) .
$$

Since the equivalent noise $\mathbf{n}_{l}$ at the $l$ th destination node is non-white, we need to perform the prewhitening operation before we swap the roles of the transmitting and the receiving nodes. After the 
pre-whitening and receive beamforming operations, the received signal at the $l$ th destination node can be expressed as

$$
\begin{aligned}
\tilde{y}_{\mathrm{d}, l} & =\mathbf{w}_{l}^{H} \mathbf{C}_{l}^{-\frac{1}{2}} \tilde{\mathbf{G}}_{l} \mathbf{F}\left(\sum_{m=1}^{L} \tilde{\mathbf{H}}_{m} \mathbf{b}_{m} s_{m}+\tilde{\mathbf{n}}_{r}\right)+\mathbf{w}_{l}^{H} \mathbf{C}_{l}^{-\frac{1}{2}} \mathbf{n}_{\mathrm{d}, l} \\
& =\mathbf{w}_{l}^{H}\left(\sum_{m=1}^{L} \overline{\mathbf{G}}_{m, l} \tilde{\mathbf{b}}_{m} s_{m}+\mathbf{C}_{l}^{-\frac{1}{2}} \tilde{\mathbf{G}}_{l} \mathbf{F} \tilde{\mathbf{n}}_{r}+\mathbf{C}_{l}^{-\frac{1}{2}} \mathbf{n}_{\mathrm{d}, l}\right), \quad l=1, \cdots, L
\end{aligned}
$$

where $\tilde{\mathbf{H}}_{l} \triangleq\left[\mathbf{H}_{1, l}^{T}, \cdots, \mathbf{H}_{K, l}^{T}\right]^{T}$ is the equivalent MIMO channel between the $l$ th source node and all relay nodes and $\overline{\mathbf{G}}_{m, l} \triangleq \mathbf{C}_{l}^{-\frac{1}{2}} \tilde{\mathbf{G}}_{l} \mathbf{F} \tilde{\mathbf{H}}_{m}\left(\mathbf{Q}_{m}+\mathbf{I}_{N_{\mathrm{s}, m}}\right)^{-\frac{1}{2}}$.

It can be seen from (22) that the equivalent noise is now white, and the received SINR in the $l$ th virtual link (where $\tilde{\mathbf{b}}_{l}^{*}$ is the receive beamforming vector and $\mathbf{w}_{l}^{*}$ becomes the transmit beamforming vector) can be expressed as

$$
\tilde{\Gamma}_{l}=\frac{\tilde{p}_{l} \tilde{\mathbf{b}}_{l}^{T} \boldsymbol{\xi}_{l l} \boldsymbol{\xi}_{l l}^{H} \tilde{\mathbf{b}}_{l}^{*}}{\sum_{m \neq l}^{L} \tilde{p}_{m} \tilde{\mathbf{b}}_{l}^{T} \boldsymbol{\xi}_{m l} \boldsymbol{\xi}_{m l}^{H} \tilde{\mathbf{b}}_{l}^{*}+\tilde{\mathbf{b}}_{l}^{T} \tilde{\mathbf{b}}_{l}^{*}}, \quad l=1, \cdots, L .
$$

Here $\boldsymbol{\xi}_{m l} \triangleq \overline{\mathbf{G}}_{l, m}^{T} \mathbf{w}_{m}^{*}, \tilde{p}_{l}$ is the transmit power in the $l$ th virtual link. Note that since the noise in the original link is pre-whitened before we swap the roles of transmitters and receivers, the equivalent virtual link noise is also white with unit-variance. Thus, the corresponding noise power after the receive beamforming is given by $\tilde{\mathbf{b}}_{l}^{T} \tilde{\mathbf{b}}_{l}^{*}$ in (23).

The optimal $\left\{\tilde{\mathbf{b}}_{l}^{*}\right\}$ can be obtained from (23) by solving the following problem for each $l=1, \cdots, L$

$$
\begin{aligned}
\min _{\tilde{\mathbf{b}}_{l}} & \sum_{m \neq l}^{L} \tilde{p}_{m} \tilde{\mathbf{b}}_{l}^{T} \boldsymbol{\xi}_{m l} \boldsymbol{\xi}_{m l}^{H} \tilde{\mathbf{b}}_{l}^{*}+\tilde{\mathbf{b}}_{l}^{T} \tilde{\mathbf{b}}_{l}^{*} \\
\text { s.t. } & \tilde{\mathbf{b}}_{l}^{T} \boldsymbol{\xi}_{l l}=1 .
\end{aligned}
$$

The solution to this problem is given by

$$
\tilde{\mathbf{b}}_{l}^{*}=\frac{\Theta_{l}^{-1} \boldsymbol{\xi}_{l l}}{\boldsymbol{\xi}_{l l}^{H} \boldsymbol{\Theta}_{l}^{-1} \boldsymbol{\xi}_{l l}}
$$

where $\boldsymbol{\Theta}_{l} \triangleq \sum_{m \neq l}^{L} \tilde{p}_{m} \boldsymbol{\xi}_{m l} \boldsymbol{\xi}_{m l}^{H}+\mathbf{I}_{N_{\mathrm{s}, l}}$ is the noise-plus-interference covariance matrix at the $l$ th receiver of the virtual link. The transmit power of the virtual link can be obtained as

$$
\tilde{\mathbf{p}} \triangleq\left(\mathbf{I}_{L}-\breve{\mathbf{G}}\right)^{-1} \tilde{\mathbf{u}}
$$

where $[\breve{\mathbf{G}}]_{l, m}=\left\{\begin{array}{ll}0, & m=l \\ \gamma_{l} \tilde{\mathbf{b}}_{l}^{T} \boldsymbol{\xi}_{m l} \boldsymbol{\xi}_{m l}^{H} \tilde{\mathbf{b}}_{l}^{*} /\left(\tilde{\mathbf{b}}_{l}^{T} \boldsymbol{\xi}_{l l} \boldsymbol{\xi}_{l l}^{H} \tilde{\mathbf{b}}_{l}^{*}\right), & m \neq l\end{array}\right.$, and $[\tilde{\mathbf{u}}]_{l} \triangleq \frac{\gamma_{l} \tilde{\mathbf{b}}_{l}^{T} \tilde{\mathbf{b}}_{l}^{*}}{\tilde{\mathbf{b}}_{l}^{T} \boldsymbol{\xi}_{l l} \boldsymbol{\xi}_{l l}^{H} \tilde{\mathbf{b}}_{l}^{*}}, l=1, \cdots, L$. Here for a vector $\mathbf{v},[\mathbf{v}]_{l}$ stands for the $l$ th element of $\mathbf{v}$. 


\section{Relay Beamforming}

In this subsection we optimize the relay amplifying matrices such that the total relay transmit power is minimized while satisfying the SINR constraints in (10). First, (4) can be rewritten as

$$
\begin{aligned}
\mathrm{E}\left\{y_{\mathrm{d}, l} y_{\mathrm{d}, l}^{*}\right\} & =\sum_{m=1}^{L} p_{m} \operatorname{tr}\left(\tilde{\mathbf{G}}_{l}^{H} \mathbf{w}_{l} \mathbf{w}_{l}^{H} \tilde{\mathbf{G}}_{l} \mathbf{F} \tilde{\mathbf{h}}_{m} \tilde{\mathbf{h}}_{m}^{H} \mathbf{F}^{H}\right)+\sigma_{\mathrm{n}}^{2} \operatorname{tr}\left(\tilde{\mathbf{G}}_{l}^{H} \mathbf{w}_{l} \mathbf{w}_{l}^{H} \tilde{\mathbf{G}}_{l} \mathbf{F} \mathbf{F}^{H}\right)+\sigma_{\mathrm{n}}^{2} \mathbf{w}_{l}^{H} \mathbf{w}_{l} \\
& =\sum_{m=1}^{L} \operatorname{tr}\left(\mathbf{R}_{\mathrm{g}, l} \mathbf{F} \mathbf{R}_{\mathrm{h}, m} \mathbf{F}^{H}\right)+\sigma_{\mathrm{n}}^{2} \operatorname{tr}\left(\mathbf{R}_{\mathrm{g}, l} \mathbf{F} \mathbf{F}^{H}\right)+\sigma_{\mathrm{n}}^{2} \mathbf{w}_{l}^{H} \mathbf{w}_{l}, \quad l=1, \cdots, L
\end{aligned}
$$

where $\mathbf{R}_{\mathrm{g}, l} \triangleq \tilde{\mathbf{G}}_{l}^{H} \mathbf{w}_{l} \mathbf{w}_{l}^{H} \tilde{\mathbf{G}}_{l}, l=1, \cdots, L$, and $\mathbf{R}_{\mathrm{h}, m} \triangleq p_{m} \tilde{\mathbf{h}}_{m} \tilde{\mathbf{h}}_{m}^{H}, m=1, \cdots, L$. Using (28), the SINR of the $l$ th link in (5) can be expressed as

$$
\Gamma_{l}=\frac{\operatorname{tr}\left(\mathbf{R}_{\mathrm{g}, l} \mathbf{F} \mathbf{R}_{\mathrm{h}, l} \mathbf{F}^{H}\right)}{\operatorname{tr}\left(\mathbf{R}_{\mathrm{g}, l} \mathbf{F}\left(\sum_{m \neq l}^{L} \mathbf{R}_{\mathrm{h}, m}+\sigma_{\mathrm{n}}^{2} \mathbf{I}_{\bar{N}_{\mathrm{r}}}\right) \mathbf{F}^{H}\right)+\sigma_{\mathrm{n}}^{2} \mathbf{w}_{l}^{H} \mathbf{w}_{l}}, \quad l=1, \cdots, L .
$$

Applying the fact that $\operatorname{tr}\left(\mathbf{A}^{H} \mathbf{B A C}\right)=\operatorname{vec}(\mathbf{A})^{H}\left(\mathbf{C}^{T} \otimes \mathbf{B}\right) \operatorname{vec}(\mathbf{A})$ [17], where $\boldsymbol{\otimes}$ denotes the matrix Kronecker product, (29) can be expressed as

$$
\Gamma_{l}=\frac{\operatorname{vec}(\mathbf{F})^{H}\left(\mathbf{R}_{\mathrm{h}, l}^{T} \otimes \mathbf{R}_{\mathrm{g}, l}\right) \operatorname{vec}(\mathbf{F})}{\operatorname{vec}(\mathbf{F})^{H}\left(\tilde{\mathbf{R}}_{\mathrm{h}, l}^{T} \otimes \mathbf{R}_{\mathrm{g}, l}\right) \operatorname{vec}(\mathbf{F})+\sigma_{\mathrm{n}}^{2} \mathbf{w}_{l}^{H} \mathbf{w}_{l}}, \quad l=1, \cdots, L
$$

where $\tilde{\mathbf{R}}_{\mathrm{h}, l} \triangleq \sum_{m \neq l}^{L} \mathbf{R}_{\mathrm{h}, m}+\sigma_{\mathrm{n}}^{2} \mathbf{I}_{\bar{N}_{\mathrm{r}}}$. Let us now introduce the link between $\mathbf{f}$ in (8) and $\operatorname{vec}(\mathbf{F})$ as $\operatorname{vec}(\mathbf{F})=\mathbf{D}_{\mathrm{F}} \mathbf{f}$, where $\mathbf{D}_{\mathrm{F}} \in \mathcal{R}^{\bar{N}_{\mathrm{r}}^{2} \times \tilde{N}_{\mathrm{r}}}$ is a matrix of ones and zeros and is constructed by observing the nonzero entries of $\operatorname{vec}(\mathbf{F})$. Note that $\mathbf{D}_{\mathrm{F}}$ does not depend on the exact numeric value of $\operatorname{vec}(\mathbf{F})$, instead it depends on the way the entries of $\mathbf{f}$ are taken to form $\operatorname{vec}(\mathbf{F})$. As an example, for a system with two relay nodes each having two antennas, there is $\mathbf{F}=\left[\begin{array}{ll}\mathbf{F}_{1} & \mathbf{0}_{2 \times 2} \\ \mathbf{0}_{2 \times 2} & \mathbf{F}_{2}\end{array}\right]$ with $\mathbf{F}_{1}=\left[\mathbf{f}_{1,1}, \mathbf{f}_{1,2}\right]$ and $\mathbf{F}_{2}=\left[\mathbf{f}_{2,1}, \mathbf{f}_{2,2}\right]$, where $\mathbf{f}_{i, j}, i, j=1,2$, are $2 \times 1$ vectors and $\mathbf{0}_{m \times n}$ denotes an $m \times n$ matrix with all zero elements. In this case, we have

$$
\operatorname{vec}(\mathbf{F})=\left[\mathbf{f}_{1,1}^{T}, \mathbf{0}_{1 \times 2}, \mathbf{f}_{1,2}^{T}, \mathbf{0}_{1 \times 2}, \mathbf{0}_{1 \times 2}, \mathbf{f}_{2,1}^{T}, \mathbf{0}_{1 \times 2}, \mathbf{f}_{2,2}^{T}\right]^{T}, \quad \mathbf{f}=\left[\mathbf{f}_{1,1}^{T}, \mathbf{f}_{1,2}^{T}, \mathbf{f}_{2,1}^{T}, \mathbf{f}_{2,2}^{T}\right]^{T}
$$

Therefore, to obtain $\operatorname{vec}(\mathbf{F})=\mathbf{D}_{\mathrm{F}} \mathbf{f}$, matrix $\mathbf{D}_{\mathrm{F}}$ should be constructed as

$$
\mathbf{D}_{\mathrm{F}}=\left(\begin{array}{llll}
\mathbf{I}_{2} & \mathbf{0}_{4 \times 2} & \mathbf{0}_{10 \times 2} & \mathbf{0}_{2 \times 2} \\
\mathbf{0}_{2 \times 2} & \mathbf{I}_{2} & \mathbf{I}_{2} & \mathbf{0}_{12 \times 2} \\
\mathbf{0}_{12 \times 2} & \mathbf{0}_{10 \times 2} & \mathbf{0}_{4 \times 2} & \mathbf{I}_{2}
\end{array}\right) .
$$

Now (30) can be rewritten as

$$
\Gamma_{l}=\frac{\mathbf{f}^{H} \mathbf{D}_{\mathrm{F}}^{T}\left(\mathbf{R}_{\mathrm{h}, l}^{T} \otimes \mathbf{R}_{\mathrm{g}, l}\right) \mathbf{D}_{\mathrm{F}} \mathbf{f}}{\mathbf{f}^{H} \mathbf{D}_{\mathrm{F}}^{T}\left(\tilde{\mathbf{R}}_{\mathrm{h}, l}^{T} \otimes \mathbf{R}_{\mathrm{g}, l}\right) \mathbf{D}_{\mathrm{F}} \mathbf{f}+\sigma_{\mathrm{n}}^{2} \mathbf{w}_{l}^{H} \mathbf{w}_{l}}, \quad l=1, \cdots, L .
$$


From (8), we have $\mathbf{f}_{k}=\mathbf{D}_{k} \mathbf{f}, k=1, \cdots, K$, with $\mathbf{D}_{k} \in \mathcal{R}^{N_{\mathrm{r}, k}^{2} \times \tilde{N}_{\mathrm{r}}}$ defined as $\mathbf{D}_{k}=\left[\mathbf{D}_{k, 1}, \cdots, \mathbf{D}_{k, K}\right]$, where $\mathbf{D}_{k, k}=\mathbf{I}_{N_{\mathrm{r}, k}^{2} \times N_{\mathrm{r}, k}^{2}}$ and $\mathbf{D}_{k, j}=\mathbf{0}_{N_{\mathrm{r}, k}^{2} \times N_{\mathrm{r}, j}^{2}}, j=1, \cdots, K, j \neq k$. By using the identity of $\operatorname{tr}\left(\mathbf{A}^{H} \mathbf{A B}\right)=\operatorname{vec}(\mathbf{A})^{H}\left(\mathbf{B}^{T} \otimes \mathbf{I}_{n}\right) \operatorname{vec}(\mathbf{A})$ for $\mathbf{A}, \mathbf{B} \in \mathcal{C}^{n \times n}$ [17], the transmit power of the $k$ th relay node in (6) can be expressed as

$$
P_{\mathrm{r}, k}=\mathbf{f}_{k}^{H}\left(\mathbf{R}_{\mathrm{y}, k}^{T} \bigotimes \mathbf{I}_{N_{\mathrm{r}, k}}\right) \mathbf{f}_{k}=\mathbf{f}^{H} \mathbf{D}_{k}^{T}\left(\mathbf{R}_{\mathrm{y}, k}^{T} \bigotimes \mathbf{I}_{N_{\mathrm{r}, k}}\right) \mathbf{D}_{k} \mathbf{f}, \quad k=1, \cdots, K
$$

Using (31) and (32), with given $\mathbf{p},\left\{\mathbf{b}_{l}\right\}$ and $\left\{\mathbf{w}_{l}\right\}$, the problem (9)-(10) can be reformulated as the following nonconvex quadratically constrained quadratic program (QCQP) problem

$$
\begin{array}{ll}
\min _{\mathbf{f}} & \mathbf{f}^{H} \mathbf{A f} \\
\text { s.t. } & \mathbf{f}^{H} \mathbf{B}_{l} \mathbf{f} \geq \gamma_{l} \sigma_{\mathrm{n}}^{2} \mathbf{w}_{l}^{H} \mathbf{w}_{l}, \quad l=1, \cdots, L
\end{array}
$$

where we introduce

$$
\mathbf{A} \triangleq \sum_{k=1}^{K} \mathbf{D}_{k}^{T}\left(\mathbf{R}_{\mathrm{y}, k}^{T} \bigotimes \mathbf{I}_{N_{\mathrm{r}, k}}\right) \mathbf{D}_{k}, \quad \mathbf{B}_{l} \triangleq \mathbf{D}_{\mathrm{F}}^{T}\left(\mathbf{R}_{\mathrm{h}, l}^{T} \bigotimes \mathbf{R}_{\mathrm{g}, l}-\gamma_{l} \tilde{\mathbf{R}}_{\mathrm{h}, l}^{T} \bigotimes \mathbf{R}_{\mathrm{g}, l}\right) \mathbf{D}_{\mathrm{F}}, \quad l=1, \cdots, L .
$$

The problem (33)-(34) is non-convex, since $\mathbf{B}_{l}$ in (35) can be indefinite. In the following, we resort to the SDR technique [18]-[21] to solve the problem (33)-(34). By introducing $\mathbf{X}=\mathbf{f} \mathbf{f}^{H}$, the problem (33)-(34) can be equivalently rewritten as

$$
\begin{array}{ll}
\min _{\mathbf{X}} & \operatorname{tr}(\mathbf{A X}) \\
\text { s.t. } & \operatorname{tr}\left(\mathbf{B}_{l} \mathbf{X}\right) \geq \gamma_{l} \sigma_{\mathrm{n}}^{2} \mathbf{w}_{l}^{H} \mathbf{w}_{l}, \quad l=1, \cdots, L \\
& \mathbf{X} \succeq 0 \\
& \operatorname{rank}(\mathbf{X})=1
\end{array}
$$

where $\mathbf{X} \succeq 0$ means that $\mathbf{X}$ is a positive semidefinite (PSD) matrix, and $\operatorname{rank}(\cdot)$ denotes the rank of a matrix. Note that in the problem (36)-(39), the cost function is linear in $\mathbf{X}$, the trace constraints are linear inequalities in $\mathbf{X}$, and the PSD matrix constraint is convex. However, the rank constraint on $\mathbf{X}$ is not convex. Interestingly, the problem (36)-(39) can be solved by the SDR technique [18]-[21] as explained in the following. First we drop the rank constraint (39) to obtain the following relaxed SDP problem which is convex in $\mathbf{X}$.

$$
\begin{array}{ll}
\min _{\mathbf{X}} & \operatorname{tr}(\mathbf{A X}) \\
\text { s.t. } & \operatorname{tr}\left(\mathbf{B}_{l} \mathbf{X}\right) \geq \gamma_{l} \sigma_{\mathrm{n}}^{2} \mathbf{w}_{l}^{H} \mathbf{w}_{l}, \quad l=1, \cdots, L \\
& \mathbf{X} \succeq 0 .
\end{array}
$$


SDP problems like (40)-(42) can be conveniently solved by using interior point methods at a complexity order that is at most $\mathcal{O}\left(\left(L+\tilde{N}_{\mathrm{r}}^{2}\right)^{3.5}\right)$ [19]. One can use, for example, the CVX MATLAB toolbox for disciplined convex programming [22] to obtain the optimal $\mathbf{X}$. Due to the relaxation, $\mathbf{X}_{\text {opt }}$ obtained by solving the problem (40)-(42) is not necessarily rank one in general. If it is, then its principal eigenvector (scaled by the square root of the principal eigenvalue of $\mathbf{X}_{\mathrm{opt}}$ ) is the optimal solution $\mathbf{f}_{\mathrm{opt}}$ to the original problem (33)-(34). If $\operatorname{rank}\left(\mathbf{X}_{\mathrm{opt}}\right) \geq 3$ and $L \leq 4$, the recent results on Hermitian matrix rank-one decomposition in [23] can be used to generate the exact optimal $\mathbf{f}_{\mathrm{opt}}$ for the problem (33)-(34) based on $\mathbf{X}_{\text {opt }}$. Otherwise, we may resort to alternative techniques such as randomization [18]-[21] to obtain a (suboptimal) $\mathbf{f}$ from $\mathbf{X}_{\mathrm{opt}}$. Different randomization techniques have been studied in the literature [18][21]. The one we choose is summarized in Table I. Note that using this approach, some of the constraints in (10) may be violated after the randomization operation. However, a feasible relay beamforming vector can be obtained by simply scaling $\mathbf{f}$ so that all the constraints are satisfied.

TABLE I

RANDOMIZATION TECHNIQUE FOR SEMIDEFINITE RELAXATION APPROACH

1) Let $\mathbf{X}=\mathbf{U} \boldsymbol{\Sigma} \mathbf{U}^{H}$ be the eigenvalue decomposition of $\mathbf{X}$.

2) Choose an $\tilde{N}_{\mathrm{r}} \times 1$ random vector $\mathbf{v}$ whose elements are independent random variables, uniformly distributed on the unit circle in the complex plane, i.e., $[\mathbf{v}]_{i}=e^{j \theta_{i}}, i=1, \cdots, \tilde{N}_{\mathrm{r}}$, where $\theta_{i}$ is independent and uniformly distributed on $[0,2 \pi)$.

3) Choose $\mathbf{f}=\mathbf{U} \boldsymbol{\Sigma}^{\frac{1}{2}} \mathbf{v}$ which ensures that $\mathbf{f}^{H} \mathbf{f}=\operatorname{tr}(\mathbf{X})$.

Now the original total transmit power minimization problem (9)-(10) can be solved by an iterative algorithm as shown in Table II. Here $\varepsilon_{i}, i=1,2$, are small positive numbers close to zero up to which convergence is acceptable, max stands for the maximal element of a vector, and the superscript $(m)$ and $[n]$ denotes the number of iterations at the outer loop and the inner loop, respectively. It can be seen from Table II that the proposed algorithm iteratively optimizes two blocks of variables: (i) The relay weighting coefficients f; (ii) The transmit beamformer vectors $\left\{\mathbf{b}_{l}\right\}$, the receive beamformer vectors $\left\{\mathbf{w}_{l}\right\}$, and the transmit power vector $\mathbf{p}$. With fixed $\mathbf{f}$, we solve the problem of optimizing $\left\{\mathbf{b}_{l}\right\},\left\{\mathbf{w}_{l}\right\}$, and $\mathbf{p}$ through step (3) in Table II. In fact, this problem is similar to the joint transceiver design problem in a single-hop MIMO interference channel [4]. Therefore, it can be shown similar to [4] that the inner iteration in step (3) converges to the optimal solution of $\left\{\mathbf{b}_{l}\right\},\left\{\mathbf{w}_{l}\right\}$, and $\mathbf{p}$ for a given $\mathbf{f}$. With fixed $\left\{\mathbf{b}_{l}\right\},\left\{\mathbf{w}_{l}\right\}$, and $\mathbf{p}$, we optimize $\mathbf{f}$ through step (2) in Table II.

In numerical simulations we observe that the outer loop converges typically within 3 to 5 iterations, 
while the inner loop converges usually within 3 iterations. However, a rigorous analysis on whether the outer loop converges to a locally optimal solution is difficult, due to the coupling between the optimization variables in (10). We also observe that the proposed algorithm requires less iterations till convergence for lower target SINR thresholds. Moreover, it can be seen from Table II that the amount of computations required for the convergence of the inner loop is much smaller than the computation involved in solving the SDP problem in the outer loop. Therefore, the overall computational complexity of the proposed algorithm can be estimated as $\mathcal{O}\left(c\left(L+\tilde{N}_{\mathrm{r}}^{2}\right)^{3.5}\right)$ with $c$ between 3 and 5.

TABLE II

PROCEDURE OF SOLVING THE PROBLEM (9)-(10) BY THE PROPOSED ITERATIVE ALGORITHM

1) Initialize the algorithm with an arbitrary forward link power vector $\mathbf{p}^{(0)}$, virtual link power vector $\tilde{\mathbf{p}}^{(0)}$, and randomly generated transmit beamforming vectors $\left\{\mathbf{b}_{l}^{(0)}\right\}$ and receive beamforming vectors $\left\{\mathbf{w}_{l}^{(0)}\right\}$; Set $m=0$.

2) Solve the subproblem (40)-(42) using known $\left\{\mathbf{b}_{l}^{(m)}\right\},\left\{\mathbf{w}_{l}^{(m)}\right\}$, and $\mathbf{p}^{(m)}$ to obtain $\mathbf{X}$.

If $\operatorname{rank}(\mathbf{X})=1$, obtain $\mathbf{f}^{(m)}$ as the principal eigenvector of $\mathbf{X}$ scaled by the square root of its principal eigenvalue.

If $\operatorname{rank}(\mathbf{X}) \geq 3$ and $L \leq 4$, use the approaches in [23] to obtain $\mathbf{f}^{(m)}$.

Otherwise

a) Use the randomization technique in Table I to obtain $\mathbf{f}$.

b) Find the most violated constraint in the original problem (9)-(10) using such $\mathbf{f}$.

c) Scale $\mathbf{f}$ so that the most violated constraint is satisfied with equality to obtain $\mathbf{f}^{(m)}$.

3) Set $n=0, \mathbf{p}^{[0]}=\mathbf{p}^{(m)},\left\{\mathbf{b}_{l}^{[0]}\right\}=\left\{\mathbf{b}_{l}^{(m)}\right\}, \tilde{\mathbf{p}}^{[0]}=\tilde{\mathbf{p}}^{(m)}$, and

a) Solve the subproblem (11)-(12) using given $\mathbf{p}^{[n]},\left\{\mathbf{b}_{l}^{[n]}\right\}$, and $\mathbf{f}^{(m)}$ to obtain $\left\{\mathbf{w}_{l}^{[n+1]}\right\}$ as in (13).

b) Solve the subproblem (14)-(15) with fixed $\mathbf{f}^{(m)},\left\{\mathbf{b}_{l}^{[n]}\right\}$, and $\left\{\mathbf{w}_{l}^{[n+1]}\right\}$ to obtain power vector $\mathbf{p}^{[n+1]}$ as in (19).

c) Update the transmit beamforming vectors $\left\{\mathbf{b}_{l}^{[n+1]}\right\}$ by solving the subproblem (24)-(25) with given $\mathbf{f}^{(m)},\left\{\mathbf{w}_{l}^{[n+1]}\right\}$, and $\tilde{\mathbf{p}}^{[n]}$.

d) Update the virtual link transmit power $\tilde{\mathbf{p}}^{[n+1]}$ with fixed $\left\{\mathbf{b}_{l}^{[n+1]}\right\},\left\{\mathbf{w}_{l}^{[n+1]}\right\}$, and $\mathbf{f}^{(m)}$ as in (27).

e) If $\max \left|\mathbf{p}^{[n+1]}-\mathbf{p}^{[n]}\right| \leq \varepsilon_{1}$, then $\mathbf{p}^{(m+1)}=\mathbf{p}^{[n+1]},\left\{\mathbf{b}_{l}^{(m+1)}\right\}=\left\{\mathbf{b}_{l}^{[n+1]}\right\},\left\{\mathbf{w}_{l}^{(m+1)}\right\}=\left\{\mathbf{w}_{l}^{[n+1]}\right\}, \tilde{\mathbf{p}}^{(m+1)}=$ $\tilde{\mathbf{p}}^{[n+1]}$; end of step 3 .

Otherwise, let $n:=n+1$ and go to step $3 \mathrm{a}$.

4) If $\max \left|\mathbf{p}^{(m+1)}-\mathbf{p}^{(m)}\right| \leq \varepsilon_{2}$, then end.

Otherwise, let $m:=m+1$ and go to step 2 .

Before moving to the next section, we would like to comment on several issues related to the implementation of the proposed algorithm in practice.

Remark 1: The channel state information (CSI) on $\left\{\mathbf{H}_{k, l}\right\} \triangleq\left\{\mathbf{H}_{k, l}, k=1, \cdots, K, l=1, \cdots, L\right\}$ and $\left\{\mathbf{G}_{l, k}\right\} \triangleq\left\{\mathbf{G}_{l, k}, l=1, \cdots, L, k=1, \cdots, K\right\}$ is required in the proposed algorithm. Since the perfect CSI is not available in a real communication system due to limited feedback and/or inaccurate channel 
estimation, robust designs can be considered in case of imperfect CSI. A worst-case based robust relay matrices design for interference relay system has been proposed in [14] where each source and destination node has a single antenna (i.e., only $\mathbf{f}$ needs to be optimized). However, when all source and destination nodes have multiple antennas, the worst-case based robust design becomes extremely challenging since the worst-case SINR $\Gamma_{l}$ is a very complicated function of $\mathbf{f},\left\{\mathbf{b}_{l}\right\},\left\{\mathbf{w}_{l}\right\}$, and $\mathbf{p}$. Alternatively, we can try the statistically robust design [24], where we average over the mismatch between the true and the estimated CSI. However, the statistical expectation of $\Gamma_{l}$ in (5) with respect to all channel matrices turns out to be an extremely complicated expression of the design variables $\mathbf{f}, \mathbf{p},\left\{\mathbf{b}_{l}\right\}$, and $\left\{\mathbf{w}_{l}\right\}$. This makes the statistically robust design problem every difficult to solve. The impact of imperfect CSI on the performance of the proposed algorithm will be studied through numerical simulation in Section IV.

Remark 2: The procedure in Table II needs to be carried out by a central processing unit due to the requirement of the global CSI. With the advancement of modern chip design, the amount of computation $\mathcal{O}\left(c\left(L+\tilde{N}_{\mathrm{r}}^{2}\right)^{3.5}\right)$ can be handled by the central processing unit. Nevertheless, it is interesting to investigate distributed algorithms that can solve the problem (9)-(10). In fact, the inner loop in step (3) of Table II is easier than step (2) for a distributed implementation. The reason is that in step (2), an SDP problem needs to be solved, which is difficult to be implemented in a distributed manner.

Remark 3: In practical applications, to meet the SINR requirements (10), some nodes may require larger transmission power that exceeds their available limit. A possible way out to this problem is to identify the SINR constraints that produce the largest increase in terms of transmit power first, and then relax those constraints in order to reduce the required power using a perturbation analysis [25]. Alternatively, one may apply an admission control algorithm first to maximize the number of links possibly served, and then perform optimal power allocation [26].

\section{NUMERiCAL EXAMPLES}

In this section, we study the performance of the proposed joint power control and beamforming algorithm for an interference MIMO relay system through numerical simulations where all nodes are equipped with multiple antennas. For simplicity, we assume $\gamma_{l}=\gamma, N_{\mathrm{s}, l}=N_{\mathrm{s}}, N_{\mathrm{d}, l}=N_{\mathrm{d}}, l=1, \cdots, L$, and $N_{\mathrm{r}, k}=N_{\mathrm{r}}, k=1, \cdots, K$, in all simulations. All noises are i.i.d. complex circularly symmetric Gaussian noise with zero mean and unit variance (i.e., $\sigma_{\mathrm{n}}^{2}=1$ ). The channel matrices have entries

generated as i.i.d. complex Gaussian random variables with zero mean and variances $\sigma_{\mathrm{h}}^{2}$ and $\sigma_{\mathrm{g}}^{2}$ for $\left\{\mathbf{H}_{k, l}\right\}$ and $\left\{\mathbf{G}_{l, k}\right\}$, respectively. All simulation results are averaged over 500 independent channel realizations.

For the proposed algorithm, the procedure in Table II is carried out in each simulation to obtain the 
power vector $\mathbf{p}$, transmit beamforming vectors $\left\{\mathbf{b}_{l}\right\}$, relay beamforming vector $\mathbf{f}$, and receive beamforming vectors $\left\{\mathbf{w}_{l}\right\}$. To initialize the algorithm in Table II, we randomly generate the transmit and receive beamforming vectors $\left\{\mathbf{b}_{l}\right\}$ and $\left\{\mathbf{w}_{l}\right\}$, respectively, along with arbitrary transmit power vector $\mathbf{p}$ and virtual power vector $\tilde{\mathbf{p}}$.

In the first example, we compare the performance of the proposed joint power control and beamforming algorithm (Proposed TxRxBF) with the relay-only beamforming without power control (RoBF-NPC) scheme studied in [13], [14] and the conventional singular-value decomposition (SVD)-based transmit beamforming approach (SVD-based TxBF). For the SVD-based TxBF scheme, we choose $\mathbf{b}_{l}$ as the principal right singular vector of $\tilde{\mathbf{H}}_{l}$. Then we update the transmit power vector $\mathbf{p}$, relay beamforming vector $\mathbf{f}$ and receive beamformers $\left\{\mathbf{w}_{l}\right\}$ based on the proposed structure. We plot the total power consumed by all source nodes and relay nodes versus the target SINR threshold $\gamma(\mathrm{dB})$. Two channel fading environments are simulated: (i) Both $\left\{\mathbf{H}_{k, l}\right\}$ and $\left\{\mathbf{G}_{l, k}\right\}$ have Rayleigh fading; (ii) Only $\left\{\mathbf{H}_{k, l}\right\}$ has Rayleigh fading while $\left\{\mathbf{G}_{l, k}\right\}$ has Ricean fading with a Ricean factor of 5. Fig. 2 shows the performance of all three algorithms for $L=2, K=15, N_{\mathrm{s}}=N_{\mathrm{r}}=2, N_{\mathrm{d}}=4, \sigma_{\mathrm{h}}^{2}=15$, and $\sigma_{\mathrm{g}}^{2}=10$. It can be seen from Fig. 2 that the proposed algorithm requires significantly less total power compared with the other two schemes in both Rayleigh and Ricean fading environments.

Note that the RoBF-NPC scheme performs better in Ricean fading channel whereas the performance of the other two approaches degrades under Ricean fading environment. This can be explained as follows. In the RoBF-NPC scheme, each transmitter and receiver has a single antenna as in [13] and [14], which indicates that the relay-destination channels $\left\{\mathbf{G}_{l, k}\right\}$ are in fact multiple-input single-output (MISO) channels. Therefore the line-of-sight (LOS) path component improves the system performance. For the other two schemes, the relay-destination channels are MIMO channels. In MIMO Ricean channels, the benefit of scattering environment reduces due to the LOS component. This weaker scattering component causes the performance degradation. Similar phenomenon has been observed in [27] for point-to-point MISO and MIMO Ricean channels.

In the second example, we vary the number of transmit antennas $N_{\mathrm{s}}$ to show the effect of transmit diversity with $L=2, K=8, N_{\mathrm{r}}=2, N_{\mathrm{d}}=4, \sigma_{\mathrm{h}}^{2}=15$, and $\sigma_{\mathrm{g}}^{2}=10$. Fig. 3 indicates the significance of transmit beamforming in the proposed algorithm. It is obvious from Fig. 3 that with the increase in the spatial dimension of the transmit beamformers the performance of the proposed algorithm keeps improving.

In the next example, we study the performance of the proposed algorithm for different number of relays $K$ with $L=2, N_{\mathrm{s}}=N_{\mathrm{r}}=2, N_{\mathrm{d}}=4, \sigma_{\mathrm{h}}^{2}=15$, and $\sigma_{\mathrm{g}}^{2}=10$. The total power required for 
$K=10,12$, and 15 versus $\gamma(\mathrm{dB})$ is displayed in Fig. 4. As expected, if we increase the number of relays the proposed algorithm requires less power since more relays provide more spatial diversity. We also show the impact of the number of relay antennas $N_{\mathrm{r}}$ in Fig. 5. This time, we set $L=3, K=12$, $N_{\mathrm{s}}=2, N_{\mathrm{d}}=4, \sigma_{\mathrm{h}}^{2}=15$, and $\sigma_{\mathrm{g}}^{2}=10$ and the total power required for $N_{r}=2$ and 3 versus $\gamma$ $(\mathrm{dB})$ is displayed. Note that with the increase in the number of relay antennas, the performance of the proposed scheme improves but at the same time, the computational complexity of solving the problem (40)-(42) significantly increases. Therefore, it is important to make a tradeoff between the performance and complexity based on the system requirements and the available resources.

In the next two examples, we study the impact of channel quality on the proposed algorithm. We assume that a larger variance of channel coefficients indicates a better channel. The impact of different $\sigma_{\mathrm{h}}^{2}$ and $\sigma_{\mathrm{g}}^{2}$ on the proposed algorithm is shown in Fig. 6 and Fig. 7, for $\sigma_{\mathrm{g}}^{2}=10$ and $\sigma_{\mathrm{h}}^{2}=10$, respectively. In these examples, we set $L=2, K=8, N_{\mathrm{s}}=N_{\mathrm{d}}=4$, and $N_{\mathrm{r}}=2$. A careful inspection of Figs. 6 and 7 reveals that the effect of channel variance of either hop is not homogeneous in general, but the results clearly demonstrate that the proposed algorithm performs better as the channel quality improves.

Next, we study the effect of channel interferences on the proposed algorithm. By increasing the number of source-destination pairs $L$, the interfering signal received at each destination node is also increased. The performance of the algorithm for different $L$ is illustrated in Fig. 8 for $K=12, N_{\mathrm{s}}=N_{\mathrm{r}}=2, N_{\mathrm{d}}=4$,

$\sigma_{\mathrm{h}}^{2}=15$, and $\sigma_{\mathrm{g}}^{2}=10$. From this figure it is clear that if there are more active users communicating simultaneously in the system, we need more power to achieve the same target SINR threshold $\gamma$.

In the last example, we study the impact of imperfect CSI on the performance of the proposed algorithm. The mismatch between the true CSI and the estimated CSI is modelled as complex Gaussian matrices with zero-mean and unit-variance entries. Fig. 9 shows the performance of all three algorithms for $L=2$, $K=12, N_{\mathrm{s}}=N_{\mathrm{r}}=2, N_{\mathrm{d}}=4, \sigma_{\mathrm{h}}^{2}=12$, and $\sigma_{\mathrm{g}}^{2}=10$. Clearly, the proposed algorithm outperforms the existing techniques with both perfect and imperfect CSI.

\section{CONCLusions}

We considered a two-hop interference MIMO relay system with distributed relay nodes and developed an iterative technique to minimize the total transmit power consumed by all source and relay nodes such that a minimum SINR threshold is maintained at each receiver. The proposed algorithm exploits beamforming techniques at the source, relay, and destination nodes in conjunction with transmit power control. Simulation results demonstrate that the proposed power control and beamforming algorithm outperforms the existing techniques. 


\section{ACKNOWLEDGMENT}

The authors would like to thank the anonymous reviewers for their valuable comments and suggestions that improve the quality of the paper.

\section{REFERENCES}

[1] D. Tse and P. Viswanath, Fundamentals of Wireless Communication. Cambridge University Press, 2005.

[2] A. Goldsmith, Wireless Communications. Cambridge University Press, 2005.

[3] F. Rashid-Farrokhi, L. Tassiulas, and K. J. R. Liu, "Joint optimal power control and beamforming in wireless networks using antenna arrays," IEEE Trans. Commun., vol. 46, pp. 1313-1324, Oct. 1998.

[4] J.-H. Chang, L. Tassiulas, and F. Rashid-Farrokhi, "Joint transmitter receiver diversity for efficient space division multiaccess," IEEE Trans. Wireless Commun., vol. 1, pp. 16-27, Jan. 2002.

[5] Y. Jing and H. Jafarkhani, "Network beamforming using relays with perfect channel information," in Proc. IEEE ICASSP, Honolulu, HI, Apr. 2007, vol. 3, pp. 473-476.

[6] A. Sendonaris, E. Erkip, and B. Aazhang, "User cooperation diversity - Part I: System description," IEEE Trans. Commun., vol. 51, pp. 1927-1938, Nov. 2003.

[7] A. Sendonaris, E. Erkip, and B. Aazhang, "User cooperation diversity - Part II: Implementation aspects and performance analysis," IEEE Trans. Commun., vol. 51, pp. 1939-1948, Nov. 2003.

[8] J. N. Laneman, D. N. C. Tse, and G. W. Wornell, "Cooperative diversity in wireless networks: Efficient protocols and outage behavior," IEEE Trans. Inf. Theory, vol. 50, pp. 3062-3080, Dec. 2004.

[9] R. Zhang, C. C. Chai, and Y.-C. Liang, "Joint beamforming and power control for multiantenna relay broadcast channel with QoS constraints,” IEEE Trans. Signal Process., vol. 57, pp. 726-737, Feb. 2009.

[10] X. Tang and Y. Hua, "Optimal design of non-regenerative MIMO wireless relays," IEEE Trans. Wireless Commun., vol. 6, pp. 1398-1407, Apr. 2007.

[11] O. Muñoz-Medina, J. Vidal, and A. Agustín, "Linear transceiver design in nonregenerative relays with channel state information," IEEE Trans. Signal Process., vol. 55, pp. 2593-2604, Jun. 2007.

[12] V. Havary-Nassab, S. Shahbazpanahi, A. Grami, and Z.-Q. Luo, "Distributed beamforming for relay networks based on second-order statistics of the channel state information," IEEE Trans. Signal Process., vol. 56, pp. 4306-4316, Sep. 2008.

[13] S. Fazeli-Dehkordy, S. Shahbazpanahi, and S. Gazor, "Multiple peer-to-peer communications using a network of relays," IEEE Trans. Signal Process., vol. 57, pp. 3053-3062, Aug. 2009.

[14] B. K. Chalise and L. Vandendorpe, "Optimization of MIMO relays for multipoint-to-multipoint communications: Nonrobust and robust designs," IEEE Trans. Signal Process., vol. 58, pp. 6355-6368, Dec. 2010.

[15] T. Taniguchi, N. B. Ramli, and Y. Karasawa, "Design of multiuser MIMO AF relay system with interference cancellation," in Proc. 6th Int. Wireless Commun. Mobile Comput. Conf., Caen, France, Jun. 2010, pp. 1075-1080.

[16] F. Rashid-Farrokhi, K. J. R. Liu, and L. Tassiulas, “Transmit beamforming and power control for cellular wireless systems," IEEE J. Select. Areas Commun., vol. 16, pp. 1437-1450, Oct. 1998.

[17] R. A. Horn and C. R. Johnson, Topics in Matrix Analysis. Cambridge, U.K.: Cambridge University Press, 1991.

[18] W.-K. Ma, T. N. Davidson, K. M. Wong, Z.-Q. Luo, and P.-C. Ching, "Quasi-ML multiuser detection using semi-definite relaxation with application to synchronous CDMA,” IEEE Trans. Signal Process., vol. 50, pp. 912-922, Apr. 2002. 
[19] N. D. Sidiropoulos, T. N. Davidson, and Z.-Q. Luo, “Transmit beamforming for physical-layer multicasting," IEEE Trans. Signal Process., vol. 54, pp. 2239-2251, Jun. 2006.

[20] S. Zhang, "Quadratic maximization and semidefinite relaxation,” Math. Program., vol. 87, ser. A, pp. 453-465, 2000.

[21] P. Tseng, "Further results on approximating nonconvex quadratic optimization by semidefinite programming relaxation," SIAM J. Optim., vol. 14, pp. 268-283, Jul. 2003.

[22] M. Grant and S. Boyd, "Cvx: Matlab software for disciplined convex programming (web page and software)." http://cvxr.com/cvx, April, 2010.

[23] W. Ai, Y. Huang, and S. Zhang, "New results on Hermitian matrix rank-one decomposition," Math. Program., ser. A (2011) 128, pp. 253-283, Aug. 2009.

[24] Y. Rong, "Robust design for linear non-regenerative MIMO relays with imperfect channel state information," IEEE Trans. Signal Process., vol. 59, pp. 2455-2460, May 2011.

[25] D. P. Palomar, M. Lagunas, and J. Cioffi, "Optimum linear joint transmit-receive processing for MIMO channels with QoS constraints," IEEE Trans. Signal Process., vol. 52, pp. 1179-1197, May 2004.

[26] K. Phan, T. Le-Ngoc, S. Vorobyov, and C. Tellambura, "Power allocation in wireless multi-user relay networks," IEEE Trans. Wireless Commun., vol. 8, pp. 2535-2545, May 2009.

[27] G. Lebrun, M. Faulkner, M. Shafi, and P. J. Smith, "MIMO Ricean channel capacity: An asymptotic analysis," IEEE Trans. Wireless Commun, vol. 5, pp. 1343-1350, Jun. 2006.

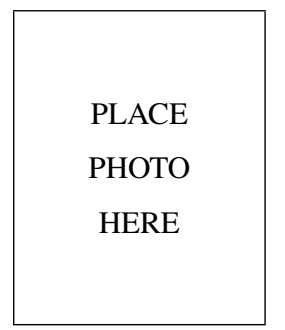

Muhammad R. A. Khandaker (S'10) received the Bachelor of Science (Honours) degree in Computer Science and Engineering from Jahangirnagar University, Dhaka, Bangladesh in 2006 and Master of Science in Telecommunications Engineering from East West University, Dhaka, Bangladesh in 2007. He is currently working toward the Ph.D. degree at the Department of Electrical and Computer Engineering, Curtin University, Australia.

Mr. Khandaker started his career as a Jr. Hardware Design Engineer in Visual Magic Corporation Limited (VMCL-BD) in November 2005. He joined as a Lecturer in the Department of Computer Science and Engineering, IBAIS University, Dhaka, Bangladesh in January 2006. In November 2007, he joined as a Lecturer in the Department of Information and Communication Technology of Mawlana Bhasani Science and Technology University, Bangladesh. Finally, he joined as a Lecturer in the Institute of Information Technology, Jahangirnagar University, Dhaka, Bangladesh in January 2008.

He was awarded Curtin International Postgraduate Research Scholarship (CIPRS) for his PhD study in 2009. He also received the Best Paper Award at the 16th Asia-Pacific Conference on Communications, Auckland, New Zealand, 2010. 
Yue Rong (S'03-M'06-SM'11) received the B.E. degree from Shanghai Jiao Tong University, Shanghai, China, the M.Sc. degree from the University of Duisburg-Essen, Duisburg, Germany, and the Ph.D. degree

PLACE (summa cum laude) from Darmstadt University of Technology, Darmstadt, Germany, all in Electrical PHOTO Engineering, in 1999, 2002, and 2005, respectively.

HERE

From February 2006 to November 2007, he was a Postdoctoral Researcher with the Department of Electrical Engineering, University of California, Riverside. Since December 2007, he has been with the Department of Electrical and Computer Engineering, Curtin University of Technology, Australia, where he is now a Senior Lecturer. His research interests include signal processing for communications, wireless communications, wireless networks, applications of linear algebra and optimization methods, and statistical and array signal processing. He has co-authored more than 80 referred IEEE journal and conference papers.

Dr. Rong received the Best Paper Award at the Third International Conference on Wireless Communications and Signal Processing, Nanjing, China, 2011, the Best Paper Award at the 16th Asia-Pacific Conference on Communications, Auckland, New Zealand, 2010, the 2010 Young Researcher of the Year Award of the Faculty of Science and Engineering at Curtin University. He is an Editor of IEEE WIRELESS COMMUNICATIONS LETTERS, a Guest Editor of the IEEE JOURNAL ON SELECTED AREAS IN COMMUNICATIONS special issue on Theories and Methods for Advanced Wireless Relays, a Guest Editor of the EURASIP JASP Special Issue on Signal Processing Methods for Diversity and Its Applications, and has served as a TPC member for IEEE ICC, WCSP, IWCMC, and ChinaCom.

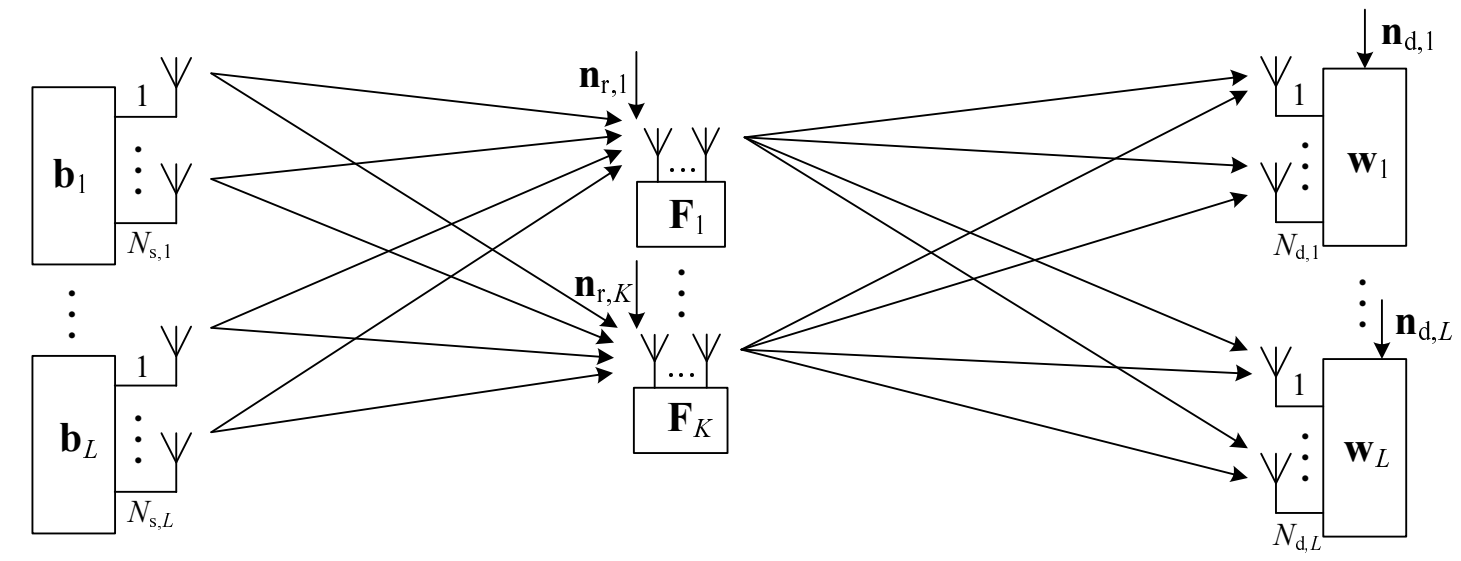

Fig. 1. Block diagram of an interference MIMO relay system. 


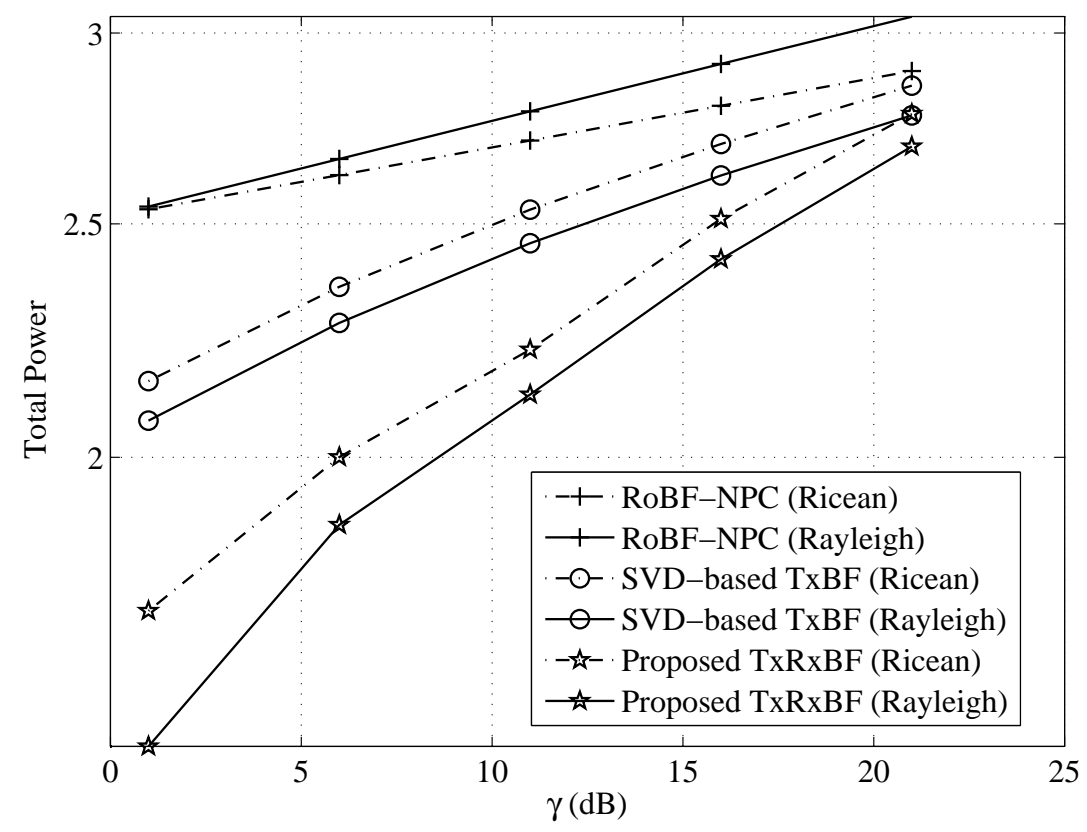

Fig. 2. Total power versus target SINR. $L=2, K=15, N_{\mathrm{s}}=N_{\mathrm{r}}=2, N_{\mathrm{d}}=4, \sigma_{\mathrm{h}}^{2}=15$, and $\sigma_{\mathrm{g}}^{2}=10$.

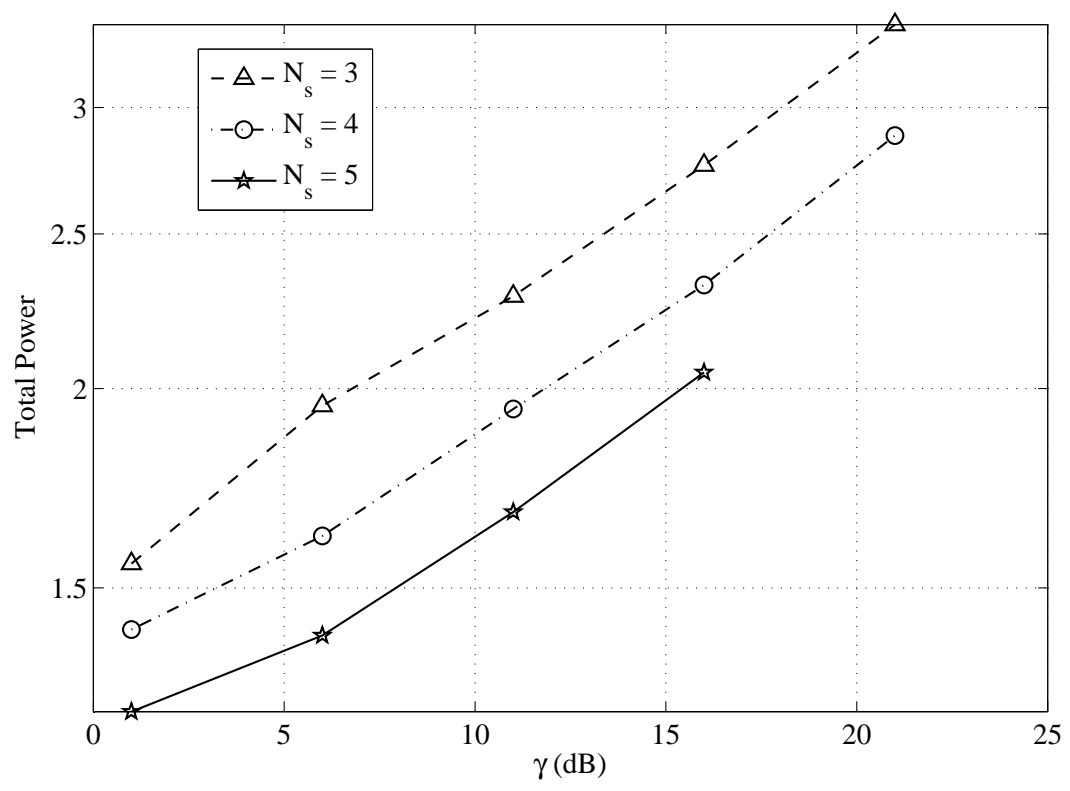

Fig. 3. Total power versus target SINR for different number of transmit antennas. $L=2, K=8, N_{\mathrm{r}}=2, N_{\mathrm{d}}=4, \sigma_{\mathrm{h}}^{2}=15$, and $\sigma_{\mathrm{g}}^{2}=10$. 


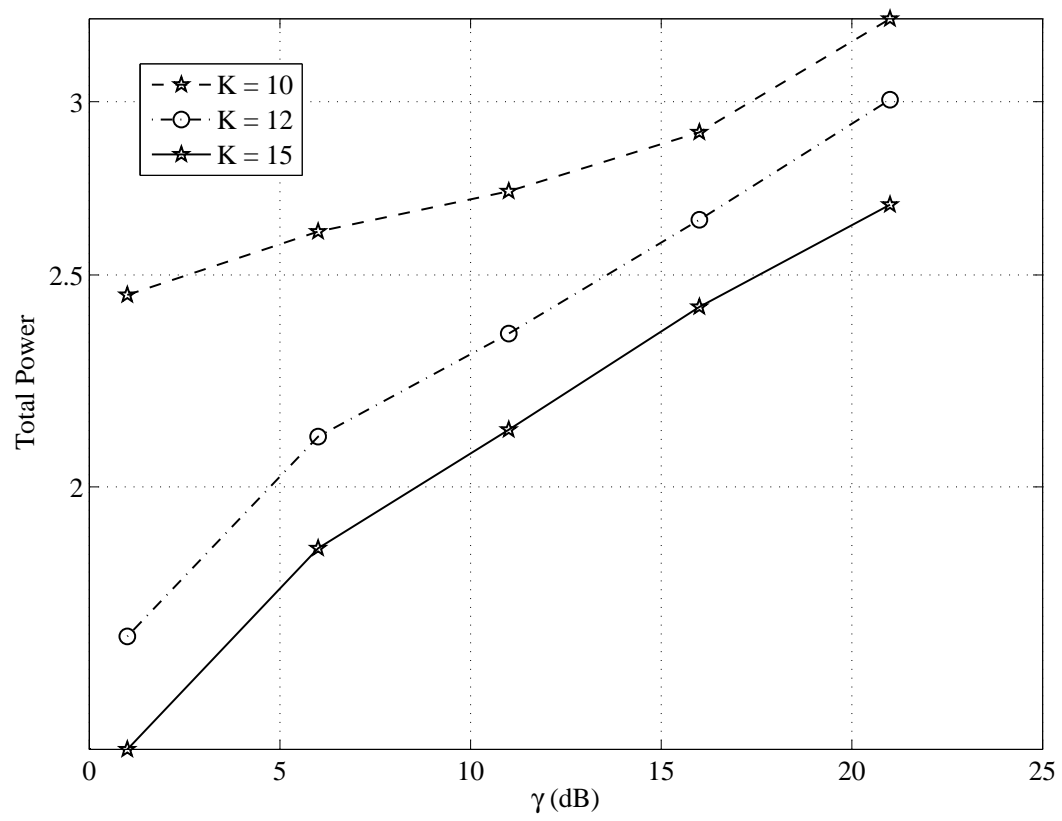

Fig. 4. Total power versus target SINR for different number of relays. $L=2, N_{\mathrm{s}}=N_{\mathrm{r}}=2, N_{\mathrm{d}}=4, \sigma_{\mathrm{h}}^{2}=15$, and $\sigma_{\mathrm{g}}^{2}=10$.

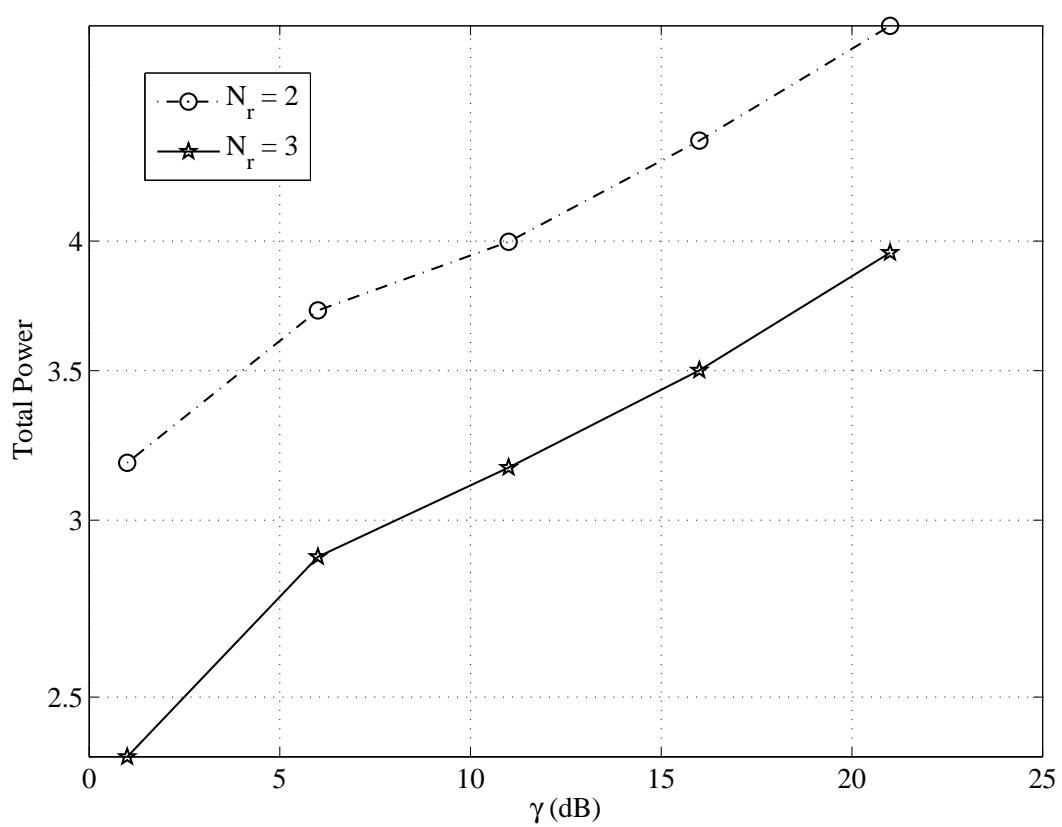

Fig. 5. Total power versus target SINR for different number of relay antennas. $L=3, K=12, N_{\mathrm{s}}=2, N_{\mathrm{d}}=4, \sigma_{\mathrm{h}}^{2}=15$, and $\sigma_{\mathrm{g}}^{2}=10$. 


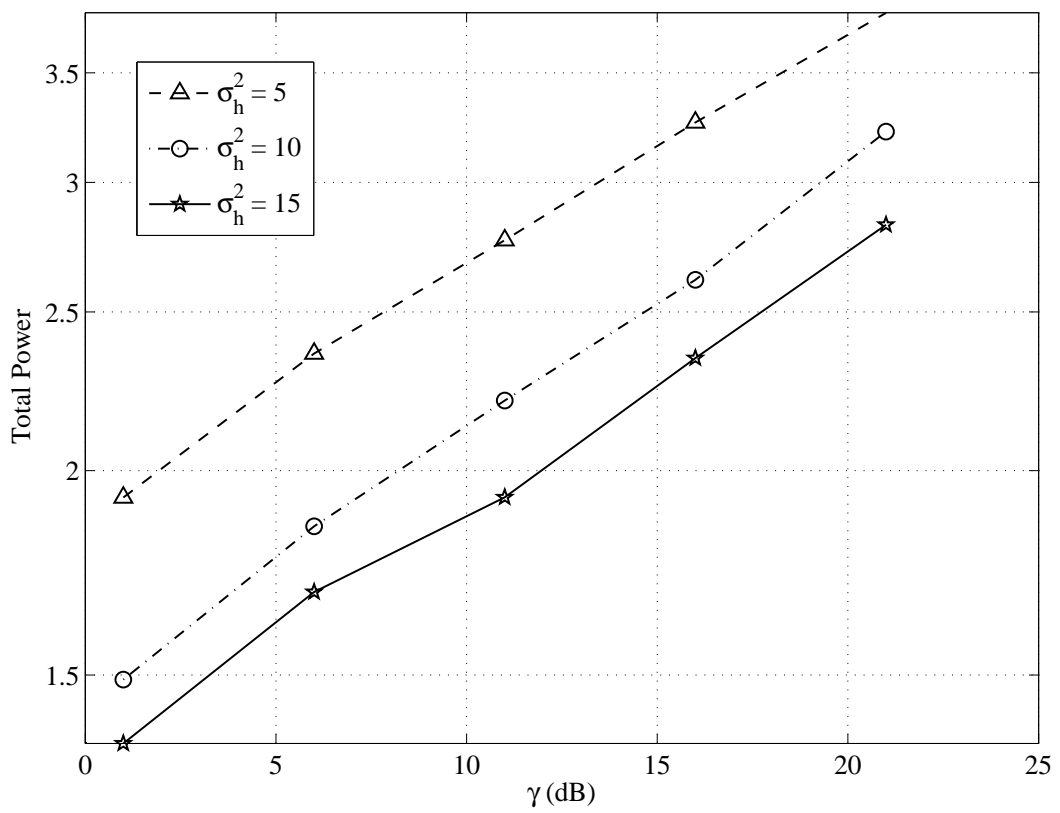

Fig. 6. Effect of the first-hop channel quality. $L=2, K=8, N_{\mathrm{s}}=N_{\mathrm{d}}=4, N_{\mathrm{r}}=2$, and $\sigma_{\mathrm{g}}^{2}=10$.

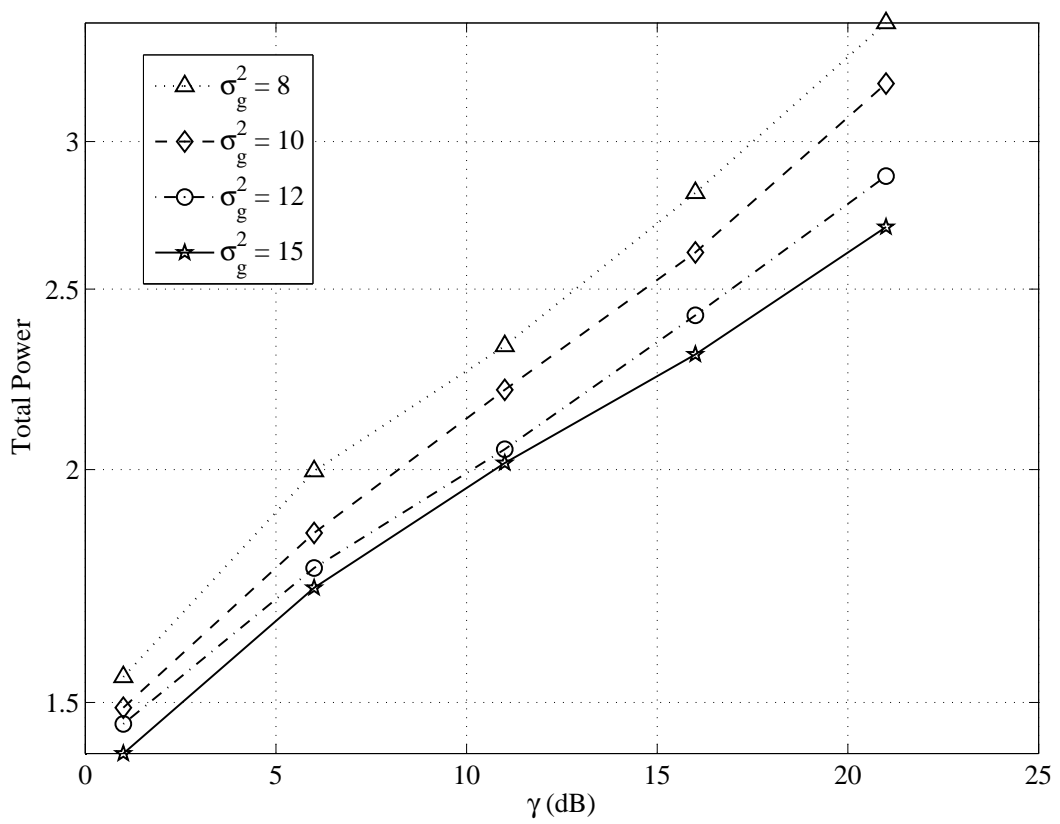

Fig. 7. Effect of the second-hop channel quality. $L=2, K=8, N_{\mathrm{s}}=N_{\mathrm{d}}=4, N_{\mathrm{r}}=2$, and $\sigma_{\mathrm{h}}^{2}=10$. 


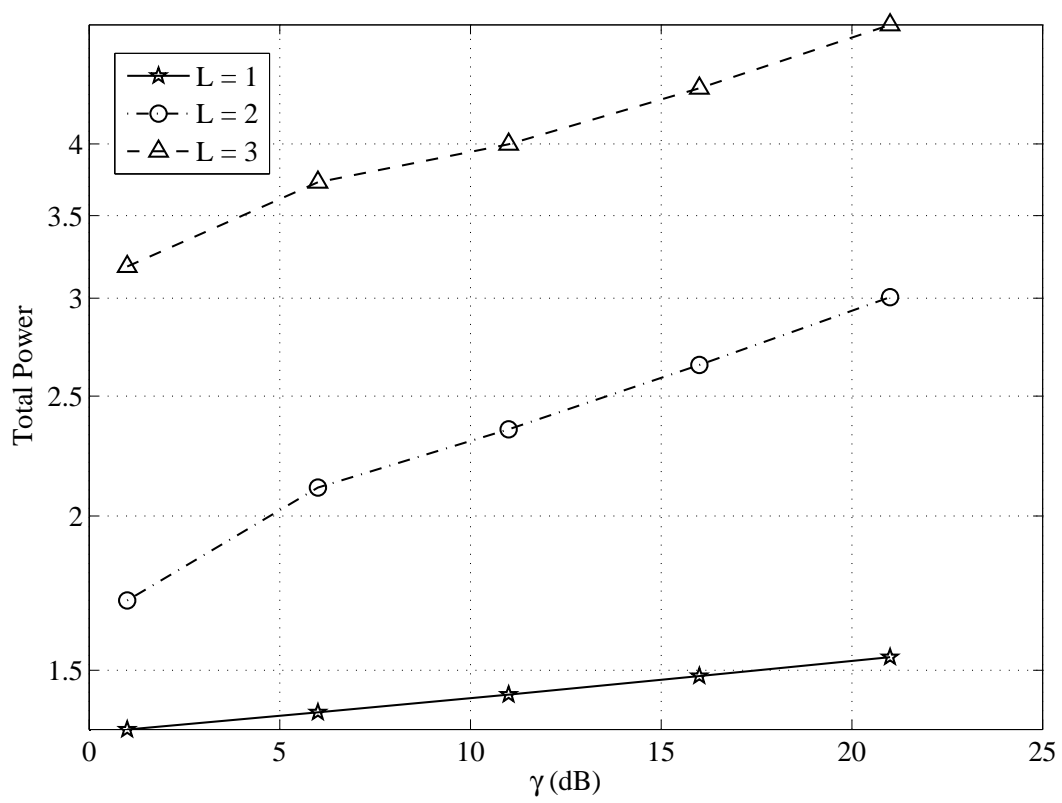

Fig. 8. Total power versus target SINR for different number of users. $K=12, N_{\mathrm{s}}=N_{\mathrm{r}}=2, N_{\mathrm{d}}=4, \sigma_{\mathrm{h}}^{2}=15$, and $\sigma_{\mathrm{g}}^{2}=10$.

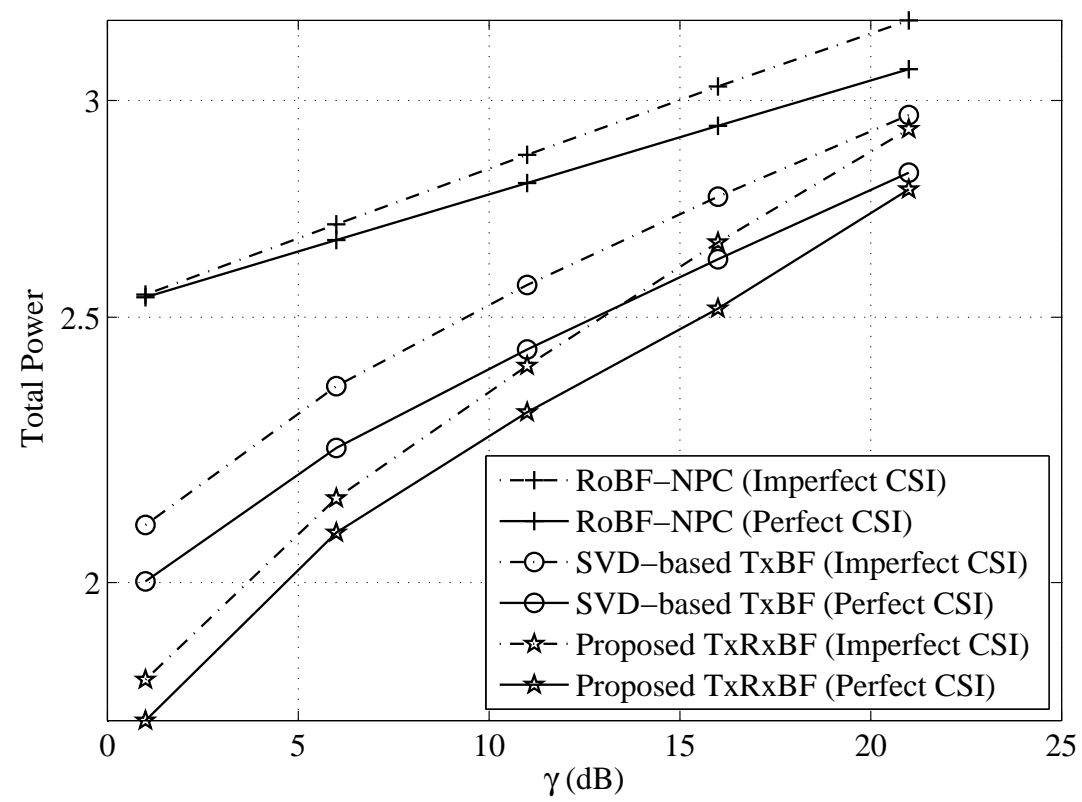

Fig. 9. The impact of the CSI mismatch on the tested algorithms. $L=2, K=12, N_{\mathrm{s}}=N_{\mathrm{r}}=2, N_{\mathrm{d}}=4, \sigma_{\mathrm{h}}^{2}=12$, and $\sigma_{\mathrm{g}}^{2}=10$. 